\title{
CUIDADOS DE ENFERMAGEM À PESSOA EM CUIDADOS PALIATIVOS COM FERIDA NEOPLÁSICA:
} REVISÃO INTEGRATIVA

\author{
NURSING CARE TO PERSON IN PALLIATIVE CARE WITH NEOPLASTIC WOUND
}

\author{
Nayara Costa Farah ${ }^{1} *$ Andyara do Carmo Pinto Coelho Paiva ${ }^{2}$ Thaís Vasconselos Amorim ${ }^{3} *$ Adélia Dayane $^{6}$ \\ Guimarães Fonseca $^{4}$ * Amanda Tamires Drumond Vilas Boas Tavares ${ }^{5} *$ Vitória Ferreira Lima $^{6} *$ Anna Maria \\ de Oliveira Salimena?
}

\begin{abstract}
RESUMO
Objetivo: conhecer os cuidados de enfermagem a pessoa que apresenta uma ferida neoplásica no contexto da assistência paliativista. Método: revisão integrativa da literatura na qual foram consultadas as bases de dados CINAHL, MEDLINE/Pubmed, LILACS e SCIELO, norteada pela questão: quais os cuidados de enfermagem a pessoa que apresenta uma ferida neoplásica no contexto da assistência paliativista? Resultados: no cuidado de enfermagem a pessoa com ferida neoplásica, observou-se que as produções científicas deram maior ênfase ao manejo de feridas, incluindo técnica de limpeza, controle do odor e da dor, coleta de material para cultura e a escolha da cobertura. Revelase escassa a produção do conhecimento acerca do apoio aos familiares e da comunicação terapêutica. Conclusão: o enfermeiro detém o conhecimento científico para tomar decisões e traçar escolhas terapêuticas para melhor assistir, no entanto o profissional deixa de perscrutar o aspecto emocional, social e espiritual, não incorporando no plano de cuidados uma abordagem integral.
\end{abstract}

Palavras-chave: Ferida; Enfermagem; Cuidados Paliativos; Neoplasias; Cuidados de Enfermagem.

\begin{abstract}
Objective: to know the nursing care of the person who presents a neoplastic wound in the context of palliative care. Method: an integrative literature review in which the CINAHL, MEDLINE / Pubmed, LILACS and SCIELO databases were consulted, guided by the question: what nursing care does the person who presents a neoplastic wound in the context of palliative care provide? Results: in nursing care for people with neoplastic wounds, it was observed that scientific productions placed greater emphasis on wound management, including cleaning techniques, odor and pain control, collection of material for culture and the choice of coverage. The production of knowledge about support for family members and therapeutic communication is scarce. Conclusion: the nurse has the scientific knowledge to make decisions and outline therapeutic choices to better assist, however the professional fails to examine the emotional, social and spiritual aspects, not incorporating a comprehensive approach in the care plan.
\end{abstract}

Keywords: Wound; Nursing; Palliative Care; Neoplasms; Nursing Care.

\footnotetext{
${ }^{1}$ Graduanda do $10^{\circ}$ período do curso de graduação em Enfermagem da Universidade Federal de Juiz de Fora - MG. Atualmente atua no projeto de extensão Atenção às Crianças Portadoras de Enurese e Disfunção do Trato Urinário Inferior. Membro do Grupo de Enfermagem e o cuidado à saúde de pessoas, famílias e coletividades em todo o ciclo vital. ORCID: https://orcid.org/0000-0003-3100-738X

${ }^{2}$ Enfermeira graduada pela Universidade Federal de Juiz de Fora (UFJF) (2010). Especialista em Políticas e Pesquisa em Saúde Coletiva pela UFJF (2011). Especialista em Saúde da Família pela UFJF, na modalidade de residência (2012). Mestre em Enfermagem PPGENF-UFJF (2014). Doutora em Enfermagem pela Escola de Enfermagem Anna Nery, UFRJ (2017). Atualmente é professora adjunta da Universidade Federal de Juiz de Fora, desenvolve atividades de ensino, pesquisa e extensão no curso de Graduação em Enfermagem e na Residência Multiprofissional em Saúde da Família. Vice- líder do Grupo de Pesquisa Enfermagem e o cuidado à saúde de pessoas, famílias e coletividades em todo o ciclo vital. ORCID: https://orcid.org/0000-0002-3567-8466

${ }^{3}$ Enfermeira graduada pela Universidade Federal de Juiz de Fora (UFJF) (2003). Especialista em Cardiologia Aplicada à Enfermagem pelo Centro de Ensino e Pesquisas do Hospital Pró-Cardíaco (2008). Mestre em Enfermagem PPGENF-UFJF (2013). Doutora em Enfermagem pela Escola de Enfermagem Anna Nery, UFRJ (2015). Atualmente é professora adjunta da Universidade Federal de Juiz de Fora, desenvolve atividades de ensino, pesquisa e extensão no curso de Graduação em Enfermagem e no Programa de Pós Graduação - Mestrado em Enfermagem. Líder do Grupo de Pesquisa Enfermagem e o cuidado à saúde de pessoas, famílias e coletividades em todo o ciclo vital. ORCID: https://orcid.org/0000-0002-7686-4839 ${ }^{4}$ Enfermeira graduada pela Universidade Estadual de Montes Claros (UNIMONTES) (2007). Especialista em Gestão Pública em Serviços de Saúde pelas Faculdades Integradas de Jacarepaguá (2010). Mestre em Ciências da Saúde PPG- UNIMONTES (2016). Doutora em Ciências da Saúde pela PPG- UNIMONTES (2018). Atualmente é professora adjunta da Universidade Federal de Juiz de Fora, desenvolve atividades de ensino, pesquisa e extensão no curso de Graduação em Enfermagem. ORCID: https://orcid.org/0000-0002-1168-7106

${ }^{5}$ Enfermeira graduada pela Universidade Federal de Viçosa (2017). Atuou como Enfermeira do Hospital do Câncer de Muriaé - Fundação Cristiano Varella, no período 2017-2019, exercendo cuidados com pacientes oncológicos clínicos, cirúrgicos e em cuidados paliativos. Mestranda em Enfermagem pela Universidade Federal de Juiz de Fora, na linha de pesquisa: Fundamentos Teóricos, Políticos e Culturais do cuidado em Saúde e Enfermagem. Bolsista da Universidade Federal de Juiz de Fora. Membro do grupo de Pesquisa Enfermagem e o cuidado à saúde de pessoas, famílias e coletividades em todo o ciclo vital. ORCID: https://orcid.org/0000-0001-9715-4599

${ }^{6}$ Graduanda do $7^{\circ}$ período do curso de graduação em Enfermagem da Universidade Federal de Juiz de Fora - MG. Atualmente é bolsista de treinamento profissional de Acompanhamento e Orientação de Estudantes do Colégio de Aplicação João XXIII da Universidade Federal de Juiz de Fora. Membro do Grupo de Enfermagem e o cuidado à saúde de pessoas, famílias e coletividades em todo o ciclo vital. ORCID: https://orcid.org/0000-0002-9140-4881

${ }^{7}$ Enfermeira graduada pela Faculdade de Enfermagem Hermantina Beraldo (1978). Especialista em Didática do Ensino Superior pela Universidade Federal de Juiz de Fora (UFJF) (1981). Especialista em Administração Universitária pela UFJF (1986). Especialista em Assistência de Enfermagem pela UFJF (1986). Especialista em Centro Cirúrgico pela Sociedade Brasileira de Enfermeiros de Centro Cirúrgico (1995). Especialista em Formação Pedagógica em Educação Profissional pela Fiocruz (2004). Mestre em Enfermagem pela Universidade Federal de Minas Gerais (2000). Doutora em Enfermagem pela Escola de Enfermagem Anna Nery, UFRJ (2007). Coordenadora do Programa de Pós-graduação Mestrado em Enfermagem da Faculdade de Enfermagem da UFJF (2010-2017) e Coordenadora Operacional do DINTER UFJF/UNICAMP de 2014-2017. Atualmente é professora titular da Universidade Federal de Juiz de Fora e desenvolve atividades no Programa de Pós Graduação - Mestrado em Enfermagem. Membro do Grupo de Pesquisa Enfermagem e o cuidado à saúde de pessoas, famílias e coletividades em todo o ciclo vital. ORCID: https://orcid.org/0000-0001$7799-665 X$
} 


\section{INTRODUÇÃO}

Segundo a Organização Mundial da Saúde (OMS), os cuidados paliativos são definidos como uma abordagem que vislumbra oferecer uma melhor qualidade de vida as pessoas que vivenciam problemas relacionados à uma doença que ameaça a continuidade da vida, assim como a seus familiares, por meio da prevenção e alívio do sofrimento através da identificação precoce, avaliação e tratamento da dor e outros problemas, sejam eles físicos, psicossociais e espirituais ${ }^{(1)}$. Recomenda-se a sua implementação $\operatorname{logo}$ no diagnóstico, intensificando na medida da necessidade. Cabe salientar que é um direito do ser humano de ser apoiado e assistido no processo de doença até a fase final da vida ${ }^{(1)}$.

Atualmente, estima-se que a cada ano, mais de 56,8 milhões de pessoas necessitam de cuidados paliativos no mundo, sendo 25,7 milhões no final da vida ${ }^{(1)}$. No Brasil, cerca de 650 mil pessoas necessitam recorrer a essa modalidade de cuidado, sendo que $80 \%$ desse número corresponde a pacientes oncológicos $^{(2)}$.

A Política Nacional para a Prevenção e Controle do Câncer na Rede de Atenção à Saúde das Pessoas com Doenças Crônicas no âmbito do Sistema Único de Saúde (SUS), publicada em maio de 2013, visa reduzir a mortalidade e a incapacidade causadas por esta doença e ainda a possibilidade de diminuir a incidência de alguns tipos de câncer, bem como contribuir para a melhoria da qualidade de vida das pessoas ${ }^{(3)}$.

Dentre as ações contempladas por essa política, destaca-se os cuidados paliativos que estão inseridos em todos os níveis de atenção na área de saúde, respeitando o conceito de hierarquização da assistência no âmbito do SUS, que se traduz na atenção básica de saúde, na média e na alta complexidades, garantindo, com isso, o direito integral, equânime e universal à saúde do cidadão ${ }^{(3)}$.

Entre os pacientes oncológicos, cerca de $5 \%$ a $10 \%$ deles desenvolvem feridas, seja em decorrência de um tumor primário ou por disseminação das células malignas ${ }^{(4)}$. Essas lesões constituem em um agravo na vida do paciente, devido sua capacidade de progressão e inviabilidade de cicatrização, progressivamente desfiguram o corpo e tornam-se dolorosas, secretivas, apresentam alto risco para infecção e miíase, podem causar sangramento e prurido, agridem o tecido saudável perilesional e liberam odor fétido $^{(5)}$.

As feridas neoplásicas são formadas pela infiltração de células malignas do tumor nas estruturas da pele, levando ao rompimento de sua integridade, decorrentes da proliferação celular descontrolada que o processo de oncogênese provoca ${ }^{(6)}$. Na fase inicial do câncer, as feridas neoplásicas são passíveis de tratamento que visam o processo de cicatrização, no entanto, quando a doença encontra em fases mais avançadas e o tratamento antineoplásico não é o mais 
indicado, o tratamento é unicamente paliativo, com intuito de controlar os sintomas físicos, psicossociais e espirituais, propiciando uma maior qualidade de vida a esse paciente ${ }^{(7)}$.

O paciente que se encontra nesse quadro, por muitas vezes sob cuidados paliativos, apresenta timidez, vergonha, baixa autoestima, isolamento, comportamento antissocial e pode não querer procurar ajuda profissional. Esse é um dos fatores que levam a piora da ferida. Outro fator é o diagnóstico tardio pelos profissionais, adiando o início do tratamento da ferida, o que leva a sua evolução ${ }^{(4,6)}$.

Desta maneira, cabe aos profissionais de saúde compreender as variadas dimensões que envolvem o cuidado com o paciente portador de ferida neoplásica, tendo em vista sua complexidade, especialmente ao profissional de enfermagem, que habitualmente é o responsável pela prevenção, a avaliação e o tratamento de feridas $^{(5)}$ além de ser um membro ativo e integrante dos cuidados paliativos ${ }^{(8)}$.

O Conselho Federal de Enfermagem respalda a prática do enfermeiro no manejo de feridas por meio da Resolução no 567/2018, visando o efetivo cuidado e segurança do paciente $^{(9)}$. Nesta perspectiva, apoiar-se em um cuidado baseado em evidências científicas para que se possa realizar o melhor tratamento à ferida, minimizando os sintomas nos quais estão incluídos o exsudato e o odor, é primordial na assistência de enfermagem ${ }^{(10)}$.
Portanto, é necessária uma avaliação criteriosa da ferida, considerando o exsudato, tamanho, configuração, órgãos e sistemas possivelmente invadidos, sangramento, tipo de dor, ou seja, um conjunto de características que irão permitir o estadiamento da lesão ${ }^{(11)}$.

Cabe ressaltar que a pessoa com ferida neoplásica deve ser atendida pelo profissional de enfermagem a partir de uma abordagem integral, uma vez que nessa condição, muitas vezes, encontra-se físico, psicológico e socialmente abalada. $\mathrm{O}$ apoio emocional ao indivíduo e sua família é primordial, abarcando outras necessidades que ultrapassam a dimensão técnica do procedimento $^{(10)}$.

Acredita-se que o presente estudo promoverá reflexões sobre como a enfermagem tem prestado a assistência na abordagem paliativista e, possivelmente, impactar no modo como se cuida da pessoa diante de uma doença progressiva e geradora de muitos conflitos, ambiguidades e medo. Este conhecimento torna-se importante ao lembrar-se que a equipe de enfermagem, constantemente, está em interação com a pessoa, seja em sua estadia no hospital, nas consultas da atenção básica e no próprio domicílio.

O objetivo deste estudo é conhecer os cuidados de enfermagem a pessoa que apresenta uma ferida neoplásica no contexto da assistência paliativista. 


\section{METODOLOGIA}

O estudo realizado trata-se de uma revisão integrativa da literatura, destacando-se como uma ferramenta que propicia a disseminação dos resultados de pesquisa sobre um determinado tema, de modo a oferecer subsídios para a prática clínica baseada em evidências. Para seu desenvolvimento as seguintes etapas foram empregadas: definição do objetivo do estudo, assim como a questão norteadora, estabelecimento dos critérios de inclusão da amostra, elucidação das informações a serem extraídas dos artigos, análise dos resultados, apresentação dos resultados e discussão e conclusão dos achados da revisão ${ }^{(12)}$.

A coleta de dados foi realizada de agosto a outubro de 2020, baseando-se na questão norteadora dessa pesquisa: quais os cuidados de enfermagem a pessoa que apresenta uma ferida neoplásica no contexto da assistência paliativista? Os artigos que não atendiam a essa questão foram automaticamente excluídos. A construção da pergunta envolveu o estudo de população e intervenção, sendo estes pontos necessários para a resolução da questão clínica pesquisada, sendo P de "população" (pessoas que apresentam uma ferida neoplásica); o I de "intervenção" (cuidados de enfermagem a esse grupo).

Por meio do acesso ao conteúdo assinado do Portal de Periódicos da CAPES (Coordenação de Aperfeiçoamento de Pessoal de Nível Superior), via login institucional da Universidade Federal de Juiz de Fora, utilizou-se as bases de dados: Cumulative Index to Nursing and Allied Health Literature (CINAHL), Medical Literature Analysis and Retrieval System Online (MEDLINE/PubMed), Literatura LatinoAmericana e do Caribe em Ciências de Saúde (LILACS) e Scientific Electronic Library Online (SCIELO). Os descritores (contidos nos Descritores em Ciências da Saúde DeCS) "ferimentos e lesões" ("wounds and injuries"), "cuidados paliativos (palliative care)" e "enfermagem" (nursing) foram combinados, por meio do conector booleano "AND". Nas bases LILACS e SCIELO, utilizou-se os descritores em português e na CINAHL e MEDLINE/PubMed, em inglês. A figura 1 apresenta a síntese das estratégias de busca utilizadas e os resultados encontrados em cada portal pesquisado. 
Figura 1- Combinação dos descritores nas bases de dados

\begin{tabular}{|c|c|c|c|c|}
\hline Descritores & Cinahl & $\begin{array}{c}\text { Medline/ } \\
\text { Pubmed }\end{array}$ & Lilacs & Scielo \\
\hline $\begin{array}{c}\text { "Ferimentos e Lesões" AND } \\
\text { "Cuidados paliativos" }\end{array}$ & - & - & 11 & 4 \\
\hline $\begin{array}{c}\text { "Ferimentos e Lesões" AND } \\
\text { "Cuidados paliativos" AND } \\
\text { "Enfermagem" }\end{array}$ & - & - & 10 & 4 \\
\hline $\begin{array}{c}\text { "Wounds and Injuries" AND } \\
\text { "Palliative care" }\end{array}$ & 95 & 1503 & - & - \\
\hline $\begin{array}{c}\text { "Wounds and Injuries" AND } \\
\text { "Palliative care" AND } \\
\text { "nursing" }\end{array}$ & 29 & 152 & - & - \\
\hline Total & 124 & 1655 & 21 & 8 \\
\hline
\end{tabular}

Fonte: Elaborado pelas autoras (2021).

Os critérios de seleção de amostras foram: evidências publicadas na literatura nacional e internacional, incluindo teses, dissertações e artigos de periódicos online com o texto disponível para acesso gratuito; produções nos idiomas português, inglês e espanhol, cujo foco tenha sido as intervenções de enfermagem no cuidado paliativo de feridas neoplásicas de adultos e idosos, na faixa etária de 19 a mais de 80 anos. O recorte temporal estabelecido foi de 15 anos, tendo em vista selecionar as evidências mais recentes. Foram excluídas resenhas, revisões de literatura, editoriais e produções duplicadas nas bases de dados.
Foram identificados, inicialmente, 1808 registros por meio da busca nos portais Lilacs, Scielo, Cinahl e Medline/PubMed. Após a leitura do título e do resumo foram excluídas as produções científicas que não estavam em consonância com o tema proposto, assim como as duplicações, elegendo 8 estudos para leitura na íntegra. Após uma análise minuciosa do material 6 artigos foram incluídos na revisão. A figura 2 apresenta o fluxograma do processo de seleção das produções científicas que constituíram a amostra deste estudo, segundo recomendação PRISMA ${ }^{(13)}$. 
Figura 2- Fluxograma representativo da busca nas bases de dados Lilacs, Scielo, Medline/Pubmed e Cinahl, 2005-2020.
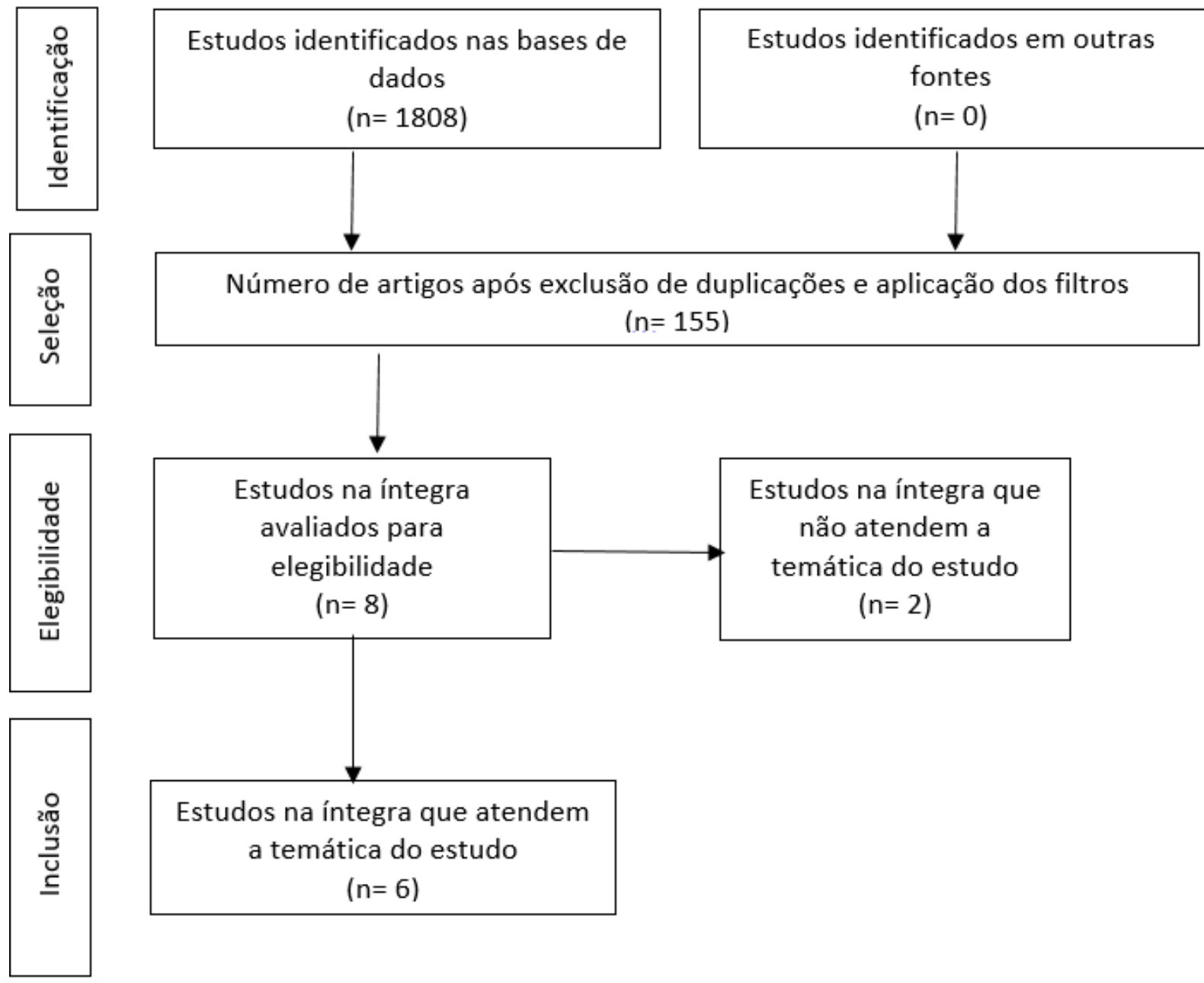

Fonte: As autoras (2021) adaptado de Galvão e colaboradores (2015) ${ }^{(13)}$.

Para categorizar nível de evidência, baseouse nas recomendações da Agency for Health care Research and Quality (AHRQ), em sete níveis de classificação: nível 1, revisão sistemática ou metanálise de ensaios clínicos controlados; nível 2, ensaio clínico controlado randomizado bem delineado; nível 3, ensaio clínico controlado sem randomização; nível 4, estudos de coorte ou caso-controle bem delineados; nível 5, revisão sistemática de estudos qualitativos e descritivos; nível 6, estudos descritivos ou qualitativos; e nível 7, opinião de autoridades ou especialistas ${ }^{(14)}$.

Para extrair e organizar os dados, assim como facilitar a caracterização, análise e discussão das publicações, utilizou-se um quadro com a descrição das seguintes informações: título; autores; ano da publicação; periódico; população e cenário de estudo; desenho do estudo e método; objetivo do estudo; resultado; implicações; nível de evidência. 


\section{RESULTADOS}

\section{As publicações que compõem essa} revisão foram codificadas com a letra "E" de estudo e numeradas de forma aleatória, para melhor organização dos resultados e facilitar a compreensão. Na figura 3 é possível acessar as publicações que constituem essa revisão com os principais resultados encontrados e o respectivo nível de evidência.

Figura 3 -Publicações que compuseram a revisão integrativa

\begin{tabular}{|c|c|c|c|c|}
\hline Código & Título/Ano & $\begin{array}{c}\text { Base de } \\
\text { dados/Periódico }\end{array}$ & $\begin{array}{c}\text { Tipo de } \\
\text { estudo/nível } \\
\text { de evidência }\end{array}$ & Resultados \\
\hline $\mathbf{E 1}^{(15)}$ & $\begin{array}{l}\text { Current practice } \\
\text { in the } \\
\text { management of } \\
\text { wound odour: An } \\
\text { international } \\
\text { survey (2014) }\end{array}$ & $\begin{array}{l}\text { CINAHL } \\
\text { International } \\
\text { Journal of } \\
\text { Nursing Studies } \\
\text { (Irlanda) }\end{array}$ & $\begin{array}{l}\text { Estudo } \\
\text { descritivo } \\
\text { qualitativo } \\
\text { (Nível 6) }\end{array}$ & $\begin{array}{l}\text { Os curativos utilizados foram: carvão, curativos à base } \\
\text { de prata. Os Agentes antimicrobianos são considerados } \\
\text { os mais eficazes, mas não foram os mais utilizados. Já } \\
\text { o metronidazol foi utilizado por } 56,9 \% \text {. Para controle } \\
\text { do odor foram utilizados uma variedade de curativos e } \\
\text { óleos de aromaterapia. }\end{array}$ \\
\hline$E 2^{(16)}$ & $\begin{array}{l}\text { Cuidados } \\
\text { paliativos de } \\
\text { enfermagem a } \\
\text { paciente com } \\
\text { carcinoma } \\
\text { espinocelular de } \\
\text { boca: um estudo } \\
\text { de caso clínico } \\
(2016)\end{array}$ & $\begin{array}{l}\text { CINAHL } \\
\text { Revista de } \\
\text { enfermagem } \\
\text { UFPE On Line } \\
\text { (Brasil) }\end{array}$ & $\begin{array}{l}\text { Estudo de } \\
\text { caso clínico } \\
\text { (Nível 7) }\end{array}$ & $\begin{array}{l}\text { Têm-se como efeito anestésico a limpeza com } \\
\text { espátulas envolvidas em gazes embebidas em colutório } \\
\text { de Cloridrato de Benzidamina e para limpeza oral a } \\
\text { escolha foi a Tirotricina associada a Quinosol } \\
\text { (Malvatricin) (ações antissépticas, antibacteriana, } \\
\text { antifúngica, propriedades desodorantes). Realizou-se a } \\
\text { extração manual de larvas e como coberturas utilizou- } \\
\text { se compressas e/ou gazes embebidas de chá de } \\
\text { camomila. Houve, também, orientação quanto ao uso } \\
\text { de opióides fortes e antidepressivos. }\end{array}$ \\
\hline$E 3^{(17)}$ & $\begin{array}{l}\text { Cuidados } \\
\text { paliativos de } \\
\text { enfermagem a } \\
\text { paciente com } \\
\text { carcinoma } \\
\text { basocelular } \\
\text { terebrante: } \\
\text { estudo de caso } \\
(2015)\end{array}$ & $\begin{array}{l}\text { CINAHL } \\
\text { Revista de } \\
\text { enfermagem } \\
\text { UFPE On Line } \\
\text { (Brasil) }\end{array}$ & $\begin{array}{l}\text { Estudo de } \\
\text { caso } \\
\text { (Nível 7) }\end{array}$ & $\begin{array}{l}\text { Alginato de cálcio foi escolhido para as áreas } \\
\text { sangrantes e muito exsudativas e metronidazol para } \\
\text { controle do odor. A limpeza do leito foi realizada com } \\
\text { jatos de solução fisiológica } 0,9 \% \text {. Além disso, } \\
\text { orientações ao paciente e familiares foram passadas, } \\
\text { como cuidados de higiene, alimentação, realização do } \\
\text { curativo e controle da dor com opióide fraco. }\end{array}$ \\
\hline $\mathbf{E} 4^{(18)}$ & $\begin{array}{l}\text { Cuidados } \\
\text { paliativos a } \\
\text { pacientes com } \\
\text { feridas } \\
\text { oncológicas em } \\
\text { hospital } \\
\text { universitário: } \\
\text { Relato de } \\
\text { experiência } \\
\text { (2014) }\end{array}$ & $\begin{array}{l}\text { LILACS } \\
\text { Revista Cogitare } \\
\text { Enfermagem } \\
\text { (Brasil) }\end{array}$ & $\begin{array}{l}\text { Relato de } \\
\text { experiência } \\
\text { (Nivel 7) }\end{array}$ & $\begin{array}{l}\text { O enfermeiro elabora um plano de cuidados pautado } \\
\text { nas características da ferida, ou seja, ensina o cuidador } \\
\text { como realiza a troca do curativo e valoriza sua } \\
\text { importância no cuidado. Além disso promove } \\
\text { orientações básicas para controle dos sintomas, oferece } \\
\text { apoio ao cuidador e identifica qual unidade de saúde } \\
\text { poderá dar continuidade aos cuidados ao paciente. }\end{array}$ \\
\hline $\mathbf{E 5}^{(19)}$ & $\begin{array}{l}\text { O perfil } \\
\text { bacteriológico e } \\
\text { as variáveis } \\
\text { relacionadas a } \\
\text { ferida neoplásica } \\
\text { no paciente em } \\
\text { cuidado paliativo } \\
\text { (2019) }\end{array}$ & $\begin{array}{l}\text { LILACS } \\
\text { Não aplica } \\
\text { (revista) } \\
\text { (Brasil) }\end{array}$ & $\begin{array}{l}\text { Estudo } \\
\text { observacional, } \\
\text { transversal e } \\
\text { descritivo } \\
\text { (Nivel 6) }\end{array}$ & $\begin{array}{l}\text { Foi realizada e indicada a coleta de cultura e análise de } \\
\text { antibiograma antes da prescrição de antibióticos } \\
\text { tópicos ou coberturas bactericidas. As coberturas } \\
\text { utilizadas foram malha de acetato de celulose, alginato } \\
\text { de cálcio, metronidazol gel, carvão ativado e } \\
\text { hemostático. }\end{array}$ \\
\hline $\mathrm{E6}^{(20)}$ & $\begin{array}{l}\text { Ocorrência e } \\
\text { manejo de } \\
\text { feridas } \\
\text { neoplásicas em } \\
\text { mulheres com } \\
\text { câncer de mama } \\
\text { avançado (2014) }\end{array}$ & $\begin{array}{l}\text { SCIELO } \\
\text { Escola Anna } \\
\text { Nery Revista de } \\
\text { Enfermagem } \\
\text { (Brasil) }\end{array}$ & $\begin{array}{l}\text { Estudo } \\
\text { transversal } \\
\text { quantitativo } \\
\text { (Nivel 6) }\end{array}$ & $\begin{array}{l}\text { Os produtos mais utilizados para tratamento foram } \\
\text { sulfadiazina de prata e AGE, produtos, segundo os } \\
\text { autores, não recomendados na literatura científica. } \\
\text { Encontrou-se, também, uma lacuna no registro de } \\
\text { dados nos prontuários, como avaliação da lesão e da } \\
\text { dor com o uso de escalas e tratamentos adotados para o } \\
\text { seu alivio, quantidade de exsudato, escala para } \\
\text { avaliação do odor, cuidados e produtos e área de } \\
\text { necrose. }\end{array}$ \\
\hline
\end{tabular}

Fonte: Elaborado pelas autoras (2021). 
A amostra final compreendeu seis estudos (E1 a E6), sendo cinco brasileiros e um irlandês, revelando poucas produções internacionais sobre o tema. Quanto ao ano de publicação, destacou-se o ano de 2014 (50\%). Os anos de 2015, 2016 e 2019 tiveram uma publicação em cada (16,66\%). Quanto ao idioma, foram cinco publicações em português e uma em inglês.

Quanto ao desenho metodológico, encontrou-se dois estudos descritivos (nível 6), dois casos clínicos (nível 7), um estudo descritivo qualitativo (nível 6) e um relato de experiência (nível 7).

Os cenários dos estudos foram as Unidades de Oncologia Clínica (E2 e E3), a Unidade de Cuidados Paliativos do INCA (E1 e E5) e um Hospital Universitário Federal (E4 e E6).

No cuidado de enfermagem a pessoa com ferida neoplásica, observou-se que as produções científicas deram maior ênfase ao manejo de feridas, incluindo técnica de limpeza, controle do odor e da dor, coleta de material para cultura e a escolha da cobertura (E1, E2, E3, E4, E5 e E6). Algumas publicações contemplam o papel educativo do enfermeiro no que tange as orientações a serem fornecidas à pessoa, seus familiares e cuidadores com o propósito de prestar informações sobre os cuidados com a ferida e no controle dos sintomas (E2, E3 e E4).

Revela-se escassa a produção do conhecimento acerca do apoio aos familiares e da comunicação terapêutica como estratégia para redução dos sintomas de ansiedade, estresse e depressão, não sendo encontrada nenhuma publicação que abordasse o assunto. Observa-se uma tendência das produções em abordar o aspecto técnico no cuidado de enfermagem à pessoa com ferida neoplásica (E2, E3 e E4), de modo que centraliza a atuação do profissional no manejo da ferida e não na pessoa que tem outras dimensões da sua vida afetadas.

As evidências encontradas nas publicações originaram a seguinte categoria de análise: Cuidados de enfermagem no manejo da ferida neoplásica e na orientação a pessoa em paliação, a família e o cuidador.

\section{DISCUSSÃO}

Cuidados de enfermagem no manejo da ferida neoplásica e na orientação a pessoa em paliação, a família e o cuidador.

A enfermagem destaca-se no cuidado paliativo a pessoa com ferida neoplásica, sendo responsável por desenvolver uma assistência sistematizada que vislumbre a integralidade do ser humano, integrando conhecimento científico que possibilita, dentre outras competências, controlar sinais e sintomas como dor, odor, exsudato, necrose, sangramento, infecção e acompanhar a evolução da lesão ${ }^{(6)}$. O processo de cuidar é comprometido quando os profissionais omitem dados importantes no prontuário (E6) como a presença de dor e o tratamento para alívio, as características da lesão, a 
quantidade de exsudato e área de necrose, interferindo diretamente na qualidade da assistência prestada a pessoa com ferida neoplásica.

$\mathrm{O}$ enfermeiro tem como autonomia o cuidado de pessoas com feridas, assim como a participação na avaliação, seleção e indicação de novas tecnologias para prevenção e tratamento de pessoas com feridas e elaboração de protocolos ${ }^{(9)}$. Além disso, o profissional de enfermagem deve ser capaz de lidar com sentimentos diversos apresentados pelo paciente oncológico em cuidado paliativo, uma vez que não há perspectiva de cura, o mesmo pode apresentar sentimentos negativos com relação ao seu quadro. Assim, o suporte psicológico precisa ser ofertado para à pessoa e sua família, visando um tratamento menos doloroso e mais humanizado $^{(21)}$.

O Enfermeiro empoderado de um arcabouço teórico, baseado em evidências científicas, tem autonomia para definir um plano de cuidados singular, conforme as características da lesão ${ }^{(9)}$. A escolha da solução a ser utilizada pelo profissional na limpeza da ferida foi mencionada no E2 e E3. A solução tópica de Cloridrato de Benzidamina para limpeza e Tirotricina associada ao Quisonol (Malvatricin) para higiene oral diária foram recomendadas nos casos de ferida neoplásica bucal. Na região da face, a proposta é soro fisiológico $0,9 \%$ em jato.
Uma revisão sistemática (22) apresentou duas soluções importantes para a limpeza de feridas foram destacadas: água corrente e soro fisiológico, sendo a água corrente uma alternativa eficaz em termos de custo, já que não se encontrou evidências de que essa aumenta a infecção.

Em contrapartida, ressalta-se que a escolha da solução depende do tipo de tecido que a ferida apresenta, elaborando um algoritmo para limpeza (23). Dessa forma, utiliza-se em tecidos desvitalizados limpeza com esfregaço, solução salina $0,9 \%$ e água potável morna a $37^{\circ} \mathrm{C}$. Para feridas com esfacelo, a opção é o PVPI ou Clorexidina; e em tecido vitalizado não será realizado o esfregaço, apenas aplicação de solução salina $0,9 \%$ e água morna a $37^{\circ} \mathrm{C}$. Porém, em tecido de granulação inviável, a limpeza poderá ser feita com PVPI ou Clorexidina.

O controle dos sintomas da pessoa com ferida neoplásica está ligado diretamente à realização do curativo e a escolha da cobertura correta. O enfermeiro, sendo um profissional que se destaca nessa área, deve estar sempre atualizado sobre os tipos de coberturas disponíveis para que se possa ser assertivo em seu tratamento, visto que existe uma grande variedade de feridas a serem tratadas (24). As publicações encontradas na presente revisão descrevem os diversos tipos de coberturas que têm sido utilizados na prática clínica do Enfermeiro. Destacam-se o uso de carvão ativado (E1 e E5), alginato de cálcio (E5), curativos à base de prata $(\mathrm{E} 1, \mathrm{E} 6)$ 
e ácidos graxos essenciais (AGE) (E6). No entanto, apesar de ainda ser empregado pelos enfermeiros, o E6 não recomenda a utilização de curativos a base de prata e com AGE.

Em consonância, destaca-se que utilização dessas coberturas não é indicada para feridas neoplásicas uma vez que a finalidade delas é a cicatrização de feridas com grande potencial de infecção como queimaduras, úlceras varicosas e feridas cirúrgicas infectadas, o que não contempla a paliação de uma ferida neoplásica ${ }^{(25)}$. Da mesma forma que nos estudos encontrados nessa revisão (E1 e E5), os autores indicam o carvão ativado, alginato de cálcio e compressas ou gazes para o controle do exsudato.

A malha de acetato também emergiu no E5 como possibilidade no tratamento de feridas neoplásicas para o controle do exsudato. A malha de acetato, carvão ativado e alginato de cálcio são coberturas que viabilizam o controle da quantidade de exsudato e, consequentemente, diminui o odor exalado. Além disso, quando associada ao petrolato, a malha diminui o potencial de sangramento e a dor ao retirar o curativo ${ }^{(4)}$.

Para o controle do exsudato, o Enfermeiro também utiliza compressas e gazes. No E2, essa cobertura secundária foi associada ao chá de camomila. Segundo pesquisa com comerciantes raizeiros, a Camomila foi citada como uma planta de efeito terapêutico para o tratamento, sendo indicada para qualquer tipo de ferida por
$53,85 \%$ dos raizeiros e somente para feridas externas por $42,3 \%$. Cabe salientar que o grau de satisfação dos usuários de plantas medicinais com essa finalidade terapêutica foi de $92,3 \%{ }^{(26)}$.

Para o controle do odor que advém da ferida neoplásica, ao abrir o curativo ou mesmo ocluído, orienta-se o uso de Metronidazol (E1, E3 e E5) com aplicação diretamente na ferida. A aromaterapia (E1) é indicada para amenizar o odor no ambiente. $\mathrm{O}$ E5 ressalta a importância de realizar a coleta (swab) de cultura e análise de antibiograma do material das feridas antes da prescrição do Metronidazol e outros antibióticos orais ou tópicos para ser mais assertivo no tratamento das infecções, gerando melhores resultados no controle do odor.

Achados corroboram com as informações apresentadas nas produções dessa revisão ${ }^{(27)}$. Uma série de cuidados de enfermagem são importantes para controle do odor como desbridamento, limpeza da ferida, curativo oclusivo e uso da bolsa de drenagem. Adicionando a esses cuidados, o uso de terapia tópica com antibióticos, coleta de amostra do local da ferida e o uso de aromatizantes no ambiente também são mencionados. Coadunando com o exposto, para o controle do odor o uso de metronidazol e outros bactericidas, uso de aromatizantes, remoção do tecido necrótico e controle do exsudato por meio de coberturas não aderentes e absorventes ${ }^{(10)}$. 
O odor é um sintoma que impacta diretamente a vida da pessoa com ferida neoplásica, fazendo com que ela, por muitas vezes, isole socialmente, sinta-se deprimida, tímida e envergonhada. Por isso, é de extrema importância que o profissional de enfermagem tenha domínio teórico e prático para que seja possível amenizar esse sintoma, melhorando, consequentemente, a qualidade de vida desse indivíduo ${ }^{(28)}$.

Apesar de os artigos encontrados na presente revisão não mencionarem o uso de escalas para avaliação do odor, um estudo de revisão integrativa ${ }^{(29)}$ evidenciou ser muito importante sua aplicação na prática clínica, pois facilita o enfrentamento do sintoma e melhora a qualidade de vida da pessoa com ferida neoplásica. Nove escalas foram apresentadas, sendo apenas uma validada. A avaliação envolve desde a intensidade até a distância que o odor é exalado.

A dor como um sintoma que se faz presente de modo constante na vida da pessoa em cuidados paliativos e nas feridas neoplásicas, causa comprometimento físico, psicológico, espiritual e social ${ }^{(30)}$. Destarte, o enfermeiro tem um papel importante no controle efetivo da dor, desde a avaliação correta das queixas até o seu manejo clínico, aliviando de forma eficaz o sofrimento ${ }^{(31)}$.

Em uma pesquisa realizada com 151 participantes com câncer em um hospital de cuidados paliativos na Malásia, o relato de dor foi evidenciado em $61,6 \%$ dos pacientes durante a admissão e em 19,9\% nas últimas
24 horas, evidenciando assim, uma alta prevalência desse sintoma em pessoas que convivem com uma doença oncológica ${ }^{(32)}$. Os estudos E2 e E3 apontaram os opioides fracos e fortes como as principais escolhas terapêuticas para analgesia da pessoa que apresenta uma ferida neoplásica.

Os opioides fortes, como Morfina e Oxicodona $^{(32,33)}$, o opioide fraco, Tramadol, e Anti-inflamatórios Não Esteroidais (AINEs), Paracetamol, são indicados para os pacientes em cuidados paliativos ${ }^{(32)}$. A Metadona é o fármaco de escolha quando se trata de um opioide forte, pois existe uma diferença considerável de custo entre as medicações, sendo ela mais barata e satisfatória em seus resultados ${ }^{(33)}$.

A atuação do enfermeiro nas orientações à pessoa, família ou cuidador quanto aos cuidados com higiene, alimentação, troca de curativos (E3) e controle da dor (E2, E3), emergiu na presente revisão. Em consonância, um estudo realizado com pacientes oncológicos identificou que as necessidades frequentemente apresentadas por pacientes oncológicos estão relacionadas a alimentação, eliminação, ambiente, atividade/repouso, relações sociais/familiares, comunicação terapêutica, suporte social/espiritual e interação com a equipe de cuidado $^{(34)}$.

Outrossim, é importante conhecer sobre as formas de avaliar esses pacientes, as medidas de intervenções farmacológicas e não farmacológicas para controle e alívio de 
sintomas, assim como a capacidade de estabelecer uma comunicação eficaz no curso da doença, estendendo-se para o período de luto, de modo a apoiar e permitir que as preferências de uma pessoa para o cuidado sejam realizadas ${ }^{(35,36)}$.

Nesta perspectiva, ressalta que a sistematização da assistência de enfermagem permite ao enfermeiro o levantamento de demandas apresentadas pelo paciente e sua família, bem como a articulação e negociação com os demais membros da equipe de saúde em nome da concretização e melhorias do cuidado, constituindo uma estratégia adequada a uma prática centrada na pessoa em sua totalidade, a fim de garantir uma assistência humanizada e de qualidade ${ }^{(37)}$.

O E4 evidencia em seus resultados a elaboração de um plano de cuidados pautado nas características da ferida, realizado pelo profissional de enfermagem, que permite a inclusão do cuidador nesse processo, assim ele se sente valorizado e mais preparado para atender as necessidades da pessoa adoecida. Além disso, a publicação destaca a necessidade do trabalho em rede, em que o enfermeiro da área hospitalar deve pactuar com os profissionais da unidade de saúde a continuidade dos cuidados ao paciente.

A comunicação entre os profissionais dos diferentes níveis de atenção se faz imprescindível para um cuidado integral e individualizado. A desarticulação entre a atenção primária e es outros níveis de atenção acarreta prejuízos na continuidade da assistência, indo de encontro aos princípios do Sistema Único de Saúde. Nessa perspectiva, os enfermeiros dos três níveis de atenção devem se comunicar e construir um plano de cuidados que abarca as necessidades do indivíduo, visando uma melhor qualidade de $\operatorname{vida}^{(38)}$.

A pessoa portadora de uma ferida neoplásica convive com a mudança da identidade corporal, a estigmatização de uma doença como um mal incurável e sintomas desagradáveis como o descontrole do exsudato e odor, provocando vergonha e timidez ${ }^{(30)}$. Nesse contexto, a comunicação terapêutica e o relacionamento interpessoal do profissional de enfermagem com o paciente e a família são imprescindíveis na sua assistência. Situações de estresse e de ansiedade, principalmente nos cuidados paliativos, são comuns e o enfermeiro precisa estar preparado para fazer da escuta e da fala uma ação terapêutica ${ }^{(39)}$.

\section{CONCLUSÃO}

As pesquisas que integraram a presente revisão revelam que o enfermeiro direciona o seu cuidado principalmente para o desenvolvimento de habilidades técnicas no cuidado à pessoa com feridas neoplásicas. $\mathrm{O}$ enfoque se deu à realização de curativos e ao controle de sinais e sintomas da ferida, uma vez que se trata de feridas crônicas em que a cicatrização não se torna o objetivo final. $\mathrm{O}$ enfermeiro detém o conhecimento científico 
para tomar decisões e traçar escolhas terapêuticas para melhor assistir, atuando desde a escolha da cobertura para o tratamento da ferida, até o esclarecimento de informações e orientações à pessoa em cuidados paliativos e seus familiares

Diante dos resultados encontrados, constata-se a necessidade de estudos que abordem o cuidado de enfermagem a pessoa com feridas neoplásica em sua totalidade, considerando todos os aspectos que envolvem o ser humano. Ainda muito centrado nos procedimentos e habilidades técnicas, o profissional deixa de perscrutar o aspecto emocional, social e espiritual, não incorporando no plano de cuidados uma abordagem integral. Acredita-se que a comunicação terapêutica poderá auxiliar em sintomas de ansiedade, angústia e em outros sofrimentos psicológicos. É imprescindível que na prática clínica do enfermeiro, principalmente nos cuidados paliativos, o foco não seja a ferida neoplásica, mas o indivíduo que convive com uma doença fora de possibilidades terapêuticas.

Acredita-se que o presente estudo seja capaz de incentivar novas produções científicas a respeito do tema, visto que o número de publicações ainda é reduzido. Além disso, considera-se que a presente revisão poderá auxiliar na prática clínica do enfermeiro no cuidado a pessoa com feridas neoplásicas, entendendo a complexidade dessa assistência que possui desafios a serem sanados pelo profissional por meio do conhecimento científico.

Constitui uma limitação do presente estudo o número reduzido, principalmente em literaturas internacionais. Por conseguinte, mostram-se necessárias pesquisas futuras sobre o tema, realizadas por óticas metodológicas variadas a fim de contribuir para o esclarecimento sobre a vivência deste fenômeno e intervenções específicas para essa população.

\section{REFERÊNCIAS}

1. World Health Organization. Global Atlas of Palliative Care at the End of Life, 2020; 2 ed.; [cited em 12 ago 2020]; Available from: http://www.thewhpca.org/resources/gl obal-atlas-on-end-of-life-care

2. Silva SO, Vidal SA, Oliveira EPC, Morais GSN, Almeida LS, Silva KLB. Conhecimento, atitudes e práticas dos profissionais de saúde sobre os cuidados paliativos a pacientes oncológicos. Rev. Eletr. Acervo Saúde [Internet]. 2019; [acesso em 12 ago 2020]; 11(9). Disponível em: https://acervomais.com.br/index.php/s aude/article/view/369/405

3. Ministério da Saúde (BR). Portaria ${ }^{\circ}$ 874, de 16 de maio de 2013. Institui a Política Nacional para a Prevenção e Controle do Câncer na Rede de Atenção à Saúde das Pessoas com Doenças Crônicas no âmbito do Sistema Único de Saúde (SUS). Gabinete do Ministro, Brasília, DF. 2013; [acesso em 20 jul 2020]; Disponível em: http://bvsms.saude.gov.br/bvs/saudele gis/gm/2013/prt0874_16_05_2013.ht $\mathrm{ml}$ 
4. Silva KRM, Bontempo PSM, Reis PED, Vasques CI, Gomes IP, Simino GPR. Intervenções Terapêuticas em Feridas Tumorais: Relato de Casos. Rev. Brasil. Cancerologia [Internet]. 2015; [cited em 21 jun 2020]; 61(4):373-9. Disponível em: https://rbc.inca.gov.br/revista/index.ph $\mathrm{p} /$ revista/article/view/232

5. Agra G, Medeiros MVS, Brito DTF, Pimentel ERS, Formiga NS, Costa MML. Conhecimento e prática de enfermeiros no controle da dor de pacientes com feridas neoplásicas. Rev. Enferm. Bras [Internet]. 2019; [acesso em 2 jun 2020]; 18(1):3-11. Disponível em: https://portalatlanticaeditora.com.br/in dex.php/enfermagembrasil/article/vie w/1039/pdf

6. Aguiar RM, Da Silva GR. Os Cuidados de Enfermagem em Feridas Neoplásicas na Assistência Paliativa. Cuidados Paliativos [Internet]. 2012; [acesso em 22 jun 2020]; 11(2):82-88. Disponível em: https://www.epublicacoes.uerj.br/index.php/revistah upe/article/view/8947

7. Instituto Nacional De Câncer José Alencar Gomes Da Silva (BR). Tratamento e controle de feridas tumorais e úlceras por pressão no câncer avançado. Série Cuidados Paliativos, Rio de Janeiro, INCA. 2009; [acesso em 22 jul 2020]. Disponível

em: https://www.inca.gov.br/

8. Brito D, Agra G, Costa M. Cuidados Paliativos a Pacientes com Ferida Neoplásica: Uma Perspectiva para a Assistência de Enfermagem. Journal of Aging \& Innovation [Internet]. 2017; [acesso em 15 jul 2020]; 6(3):28-38. Disponível em: http://www.journalofagingandinnovati on.org/wp-content/uploads/3-feridaneopl\%C3\%A1sica.pdf
9. Conselho Federal De Enfermagem (BR). Resolução COFEN 567/2018, de 29 de janeiro de 2018. Regulamenta a atuação da equipe de enfermagem no cuidado aos pacientes com feridas. COFEN, Brasília, DF. 2018; [acesso em 22 jul 2020]. Disponível em: http://www.cofen.gov.br/wpcontent/uploads/2018/02/RESOLU\%C 3\%87\%C3\%83O-567-2018.pdf

10. Soares RS, Cunha DAO, Fuly PSC. Cuidados de enfermagem com feridas neoplásicas. Rev. Enferm. UFPE online [Internet]. 2019; [acesso em 18 jul 2020]; 12(12):3456-63. Disponível em:

https://periodicos.ufpe.br/revistas/revi staenfermagem/article/view/236438

11. Agra G, Costa MML. Feridas Neoplásicas. In: Campos, MGCA. et al. Feridas complexas e estomias: aspectos preventivos e manejo clínico [E-book]. João Pessoa: Editora Ideia; 2016; [acesso em 03 jun 2020]; Cap. 10, p. 303-20. Disponível em: http://www.corenpb.gov.br/3565_356 5.html

12. Souza MT, Silva MD, Carvalho R. Integrative review: what is it? How to do it? Einstein [Internet]. 2010; [cited em 03 ago 2020]; 8(1):102-6. Available from: https://www.scielo.br/pdf/eins/v8n1/1 679-4508-eins-8-1-0102.pdf

13. Galvão TF, Pansani TSA, Harrad D. Principais itens para relatar Revisões sistemáticas e Meta-análises: A recomendação PRISMA. Epidemiol. Serv. Saúde [Internet]. 2015; [acesso em 03 ago 2020]; 24(2). Disponível em:

https://www.scielo.br/pdf/ress/v24n2/ 2237-9622-ress-24-02-00335.pdf

14. Galvão CM. Evidence hierarchies. Acta Paul Enferm [Internet]. 2006; 
[cited em 03 ago 2020]; 19(2). Available from: https://www.scielo.br/scielo.php?scrip $\mathrm{t}=\mathrm{sci}$ issuetoc\&pid=0103-

210020060002\&lng=en\&nrm=iso

15. Gethin G, Grocott P, Probst S, Clarke E. Current practive in the management of wound odour: na international survey. Internat. Journal Nurs. Stud. [Internet]. 2014; [cited em 30 ago 2020]; 51(6):856-74. Available from: https://pubmed.ncbi.nlm.nih.gov/2423 8490/

16. Agra G, Gouveia BL, Sousa AT, Soares MJ, Oliveira SH, Costa MM. Cuidados paliativos de enfermagem a paciente com carcinoma espinocelular de boca: estudo de caso clínico. Rev. Enferm. UFPE online [Internet]. 2016; [acesso em 03 set 2020]; 10(6):214958. Disponível em: https://periodicos.ufpe.br/revistas/revi staenfermagem/article/view/11229

17. Agra G, Gouveia BLA, Sousa ATO, Costa MML, Oliveira SHS, Soares MJG. Cuidados paliativos de enfermagem a paciente com carcinoma basocelular terebrante: estudo de caso. Rev. Enfer. UFPE online [Internet]. 2015, [acesso em 20 set 2020]; 9(11):9873-81. Disponível em:

https://www.semanticscholar.org/pape r/CUIDADOS-PALIATIVOS-DEENFERMAGEM-A-PACIENTECOM-DE-Agra-

Lourdes/0dc922e24081fefd36fce6494 61ed9441a469558

18. Castro MCF, Cruz PS, Grellmann MS, Santos WA, Fuly PSC. Cuidados paliativos a pacientes com feridas oncológicas em hospital universitário: relato de experiência. Rev. Cogitare Enfermagem [Internet]. 2014; [acesso em 30 set 2020]; 19(4):841-44. Disponível em: https://revistas.ufpr.br/cogitare/article/ view/37294
19. Soares RS. O perfil bacteriológico e as variáveis relacionadas a ferida neoplásica no paciente em cuidado paliativo [dissertation]. Niterói: Escola de Enfermagem Aurora de Afonso Costa, Universidade Federal Fluminense; 2019 [acesso em 03 out 2020]. Disponível em: https://app.uff.br/riuff/handle/1/9596

20. Gozzo TO. Tahan FP, Andrade M, Nascimento TG, Prado MAS. Ocorrência e manejo de feridas neoplásicas em mulheres com câncer de mama avançado. Esc. Anna Nery Rev. Enferm. [Internet]. 2014; [acesso em 20 out 2020]; 18(2):270-76. Disponível em: https://www.scielo.br/pdf/ean/v18n2/1 414-8145-ean-18-02-0270.pdf

21. Santos ALM, Lira SS, Costa RSL. Cuidados paliativos prestados pelo enfermeiro ao paciente oncológico. DêCiência em Foco [Internet]. 2018; [acesso em 03 nov 2020]; 2(1):63-77. Disponível em: http://revistas.uninorteac.com.br/index .php/DeCienciaemFoco0/article/view/ 147

22. Santos E, Queirós P, Cardoso D, Cunha M, Apóstolo J. A eficácia das soluções de limpeza para o tratamento de feridas: uma revisão sistemática. Rev. Enferm. Ref. [Internet]. 2016; [acesso em 03 nov 2020]; 4(8):133144. Disponível em: https://www.researchgate.net/publicati on/303596754_A_eficacia_das_soluco es_de_limpeza_para_o_tratamento_de _feridas_uma_revisao_sistematica

23. Santos AC, Dutra RAA, Salomé GM, Ferreira LM. Construção e confiabilidade interna de um algoritmo para a escolha da limpeza e terapia tópica em feridas. Rev. Enferm. UFPE online [Internet]. 2018; [acesso em 10 nov 2020]; 12(5). Disponível em: 
https://pesquisa.bvsalud.org/portal/res ource/pt/biblio-980414

24. Prado ARA, Barreto VPM, Tonini T, Silva AS, Machado WC. O saber do enfermeiro na indicação de coberturas no cuidado ao cliente com feridas. Estima [Internet]. 2016; [acesso em 10 nov 2020]; 14(4):175-82. Disponível em:

https://www.revistaestima.com.br/inde x.php/estima/article/view/430

25. Lisboa IND, Valença MP. Caracterização de pacientes com feridas neoplásicas. Estima [Internet]. 2016; [acesso em 20 nov 2020]; 14(1):21-28. Disponível em: https://www.revistaestima.com.br/inde x.php/estima/article/view/116

26. Nascimento MWA, Veríssimo RCSS, Bastos MLA, Bernardo THL. Indicações de plantas medicinais realizadas por raizeiros para tratamento de feridas. Ver. Eletr. de Enferm. [Internet]. 2016; [acesso em 20 nov 2020]; 18. Alagoas, v. 18. $2016 . \quad$ Disponível em: https://revistas.ufg.br/fen/article/view/ 31143\#: :text=Foram $\% 20$ citadas $\% 20$ 48\%20esp\%C3\%A9cies\%20vegetais,i ndica\%C3\%A7\%C3\%A3o\%20para\% 20 uso $\% 20$ em\%20feridas

27. Castro MCF, Santos WA, Fuly PSC, Santos MLSC, Ribeiro-Garcia T. Intervenções de enfermagem para pacientes oncológicos com odor fétido em ferida tumoral. Aquichán [Internet]. 2017; [acesso em $02 \mathrm{dez}$ 2020]; 17(3). Disponível em: https://www.redalyc.org/jatsRepo/741/ 74155184002/html/index.html

28. Santos WA, Fuly PSC, Santos MLSC, Sauto MD, Reis CM, Castro MCF. Avaliação do isolamento social em pacientes com odor em feridas neoplásicas: revisão integrativa. Rev. Enferm. UFPE online [Internet]. 2017; [acesso em $03 \mathrm{dez} 2020]$; 11(3):1495-
503. N DERME https://periodicos.ufpe.br/revistas/revi staenfermagem/article/view/13995

29. Souza MAO, Souza NR, Melo JTS, Xavier MACB, Almeida GL, Santos ICRV. Escalas de avaliação de odor em feridas neoplásicas: uma revisão integrativa. Rev. Brasil. Enferm. [Internet]. 2018; [acesso em $03 \mathrm{dez}$ 2020]; 71(5). Disponível em: https://www.scielo.br/scielo.php?scrip $\mathrm{t}=$ sci_arttext\&pid=S0034-

$71672018000502552 \& \operatorname{lng}=\mathrm{en} \& \mathrm{nrm}=\mathrm{i}$ so\&tlng=pt

30. Beretta LL, Santos MLSC, Fuly PC, Berardinelli LMM. Resiliência no processo do cuidado aos pacientes com feridas tumorais malignas: revisão integrativa. Research, Society and Development [Internet]. 2020; [acesso em 15 dez 2020]; 9(4). Disponível em: https://www.researchgate.net/publicati on/340239373_Resiliencia_no_proces so_do_cuidado_aos_pacientes_com_f eridas_tumorais_malignas_revisao_int egrativa

31. Andrade FLM, Silva MES, Brito DTF, Agra G, Macedo EL, Sousa ATO. Dor oncológica: manejo clínico realizado por enfermeiros. Rev. Iniciação Cient. da Univers. Vale do Rio Verde [Internet]. 2018; [acesso em $15 \mathrm{dez}$ 2020]; 8(1): 3-16. Disponível em: http://periodicos.unincor.br/index.php/ iniciacaocientifica/article/view/4244\#: $\sim$ :text=DOR\%20ONCOL\%C3\%93GI CA\%3A\%20manejo\%20c1\%C3\%ADn ico\%20realizado\%20por\%20enfermei ros,-

32. Mejin M, Keowmani T, Rahman SA, Liew J, Lai J, Chua M, Che Wan I. Prevalence of pain and treatment outcomes among cancer patients in a Malaysian palliative care unit. Pharmacy Practice [Internet]. [cited em 05 jan 2021]; 2017; 17(1). Available from: 
https://pubmed.ncbi.nlm.nih.gov/3101 $5879 /$

33. Peirano GP, Mammana GP, Bertolino MS, Pastrana T, Vega GF, Russo J, Varela G, Vignaroli E, Ruggiero R, Armesto A, Camerano G, Dran G. Methadone as first-line opioid treatment for cancer pain in a developing country palliative care unit. Support Care Cancer [Internet]. 2016; [cited em 05 jan 2021]; 24(8):3551-56. Available from: https://pubmed.ncbi.nlm.nih.gov/2702 2964/

34. Brito KCFV, Sousa SR. As necessidades de cuidado do cliente oncológico hospitalizado: aplicação da taxonomia Nanda. Rev. Fund Care Online [Internet]. 2017; [acesso em 17 jan 2021]; 9(2):327-32. Disponível em:

http://www.seer.unirio.br/index.php/c uidadofundamental/article/view/4138

35. Yates P. Symptom Management and Palliative Care for Patients with Cancer. Nursing Clinics Of North America [Internet]. 2017; [cited em 18 jan 2021]; 52(1):179-91. Available from:

https://pubmed.ncbi.nlm.nih.gov/2818 9162/

36. Kanno Y, Sato K, Shimizu M, Funamizu Y, Andoh H, Kishino M, et al. Development and Validity of the Nursing Care Scale and Nurse's Difficulty Scale in Caring for Dying Patients With Cancer and Their Families in General Hospitals in Japan. Feature Article [Internet]. 2018; [cited em 10 fev 2021];20(6):19. Available from: https://www.ncbi.nlm.nih.gov/pubmed /30063557

37. Silva EVS, Conceição HN. Cuidados de enfermagem a pacientes com feridas oncológicas. Rev. Espaço para a Saúde [Internet]. 2020; [acesso em
25 fev 2020]; 21(1)82-94. Disponível em:

https://docs.bvsalud.org/biblioref/2020 /07/1104425/08-693-1752-2-

ed_revisado_portugues16194-1pdfcuidados-

aliati_hAKhF9N.pdf\#: :text=Os\%20c uidados $\% 20$ paliativos $\% 20 \mathrm{de} \% 20 \mathrm{enfer}$ magem $\% 20 \mathrm{em} \% 20$ pacientes $\% 20 \mathrm{com}$ $\% 20$ feridas $\% 20$ neopl\%C3\%A1 sicas,n eoplasias $\% 20$ sem $\% 20$ possibilidade $\% 2$ 0de\%20cura.

38. Moll MF, Goulart MB, Caprio AP, Ventura CAA, Ogoshi AACM. O conhecimento dos enfermeiros sobre as redes de atenção à saúde. Rev. Enferm. UFPE on line [Internet]. 2017; [acesso em 05 mar 2021]; 11(1):86-93. Disponível em: https://pesquisa.bvsalud.org/portal/res ource/pt/bde-30275

39. Campos C. A comunicação terapêutica enquanto ferramenta profissional nos cuidados de enfermagem. Rev. Serv. Psiquiat. Hosp. Prof. Dr Fernando Fonseca [Internet]. 2017; [acesso em 05 mar 2021]; 15(1):91-101. Disponível em: https://revistas.rcaap.pt/psilogos/articl e/view/9725

Autora correspondente: Andyara do Carmo Pinto Coelho Paiva Rua Ubá, 153/201, Democrata. Juiz de ForaMG. CEP: 36035-260.

Submissão: 2021-03-19

Aprovado: 2021-05-24 